\title{
Effective simulation methods for polyelectrolytes in low dielectric solvents
}

\author{
M. O. Khan* \\ G. Kennedy ${ }^{\dagger}$ \\ D. Y. C. Chan
}

(Received 22 November 2004, revised 22 October 2005)

\begin{abstract}
Simulations of polyelectrolytes in low dielectric solvents converge slowly. This can be circumvented by using clothed global moves or parallel expanded techniques. In clothed moves the counterions are moved with the polyelectrolyte backbone and are not left behind when a substantial part of the chain is moved. For moderately charged systems the speed-up has been shown to be by a factor of up to 3 . We show how, for systems with strong electrostatic interactions, much larger efficiency gains in the simulation process, by a factor of over 400, can
\end{abstract}

${ }^{*}$ Particulate Fluids Processing Centre, Dept. Mathematics \& Statistics, The University of Melbourne, Victoria, Australia. mailto:m.khan@ms.unimelb.edu.au

${ }^{\dagger}$ School of Mathematical Sciences, Monash University, Victoria, Australia. mailto: gareth. kennedy@maths.monash.edu . au

${ }^{\ddagger}$ Particulate Fluids Processing Centre, Dept. Mathematics \& Statistics, The University of Melbourne, Victoria, Australia. mailto:d.chan@ms.unimelb.edu . au

See http://anziamj.austms.org.au/V46/CTAC2004/Khan for this article, (c) Austral. Mathematical Soc. 2005. Published November 17, 2005. ISSN 1446-8735 
be obtained. In our parallel expanded algorithm, a number of conventional Monte-Carlo simulations are carried out in parallel, with only the dielectric constant being different. By allowing the different simulations to communicate and exchange conformations, trapped configurations in the low dielectric simulations may escape via the higher dielectric simulations. We show how this method scales linearly up to 8 processors for highly charged polyelectrolytes.

\section{Contents}

1 Introduction

C1255

2 The model and simple Monte-Carlo method

$\mathrm{C} 1257$

3 Clothed global moves

C1259

4 Parallel expanded ensembles

$\mathrm{C} 1264$

5 Conclusion

C1269

References

$\mathrm{C} 1270$

\section{Introduction}

The conformational behaviour of polyelectrolytes (charged polymers) have been the focus of many theoretical studies, see for example the review by Ullner [14]. In contrast to predictions of conventional mean field theories, computer simulation studies have shown that the effective interaction between like-charged monomers can be attractive when the electrostatic interactions are strong $[13,15,10]$. Here we consider polyelectrolytes immersed in low dielectric solvents such as alcohol. By mixing water and ethanol the dielectric 
constant $\epsilon$ can be varied from 78 for pure water to 24 for pure ethanol, that is, the electrostatic interactions are three times larger in ethanol compared to water. A major problem for simulations of polyelectrolytes with strong electrostatic interactions is that calculations of the conformational properties of the macromolecules tend to converge very slowly. When equilibrium properties are of interest, Monte-Carlo (MC) simulations explore the free energy landscape by employing unphysical moves, that is, moves that do not follow the actual dynamics of the system. Unfortunately, while such methods are effective for neutral polymers and polyelectrolytes that interact with the the screened coulomb potential, they do not provide the same efficiency for systems with strong electrostatic interactions when explicit counterions are used. Also, due to the long range character of the Coulomb interaction all pair-wise interactions have to be calculated and this provides a limit on the number of charged particles that can be considered in a simulation. The long range interaction also prohibits the use of standard parallel computational techniques such as domain decomposition. Here we compare in detail the performance of two different approaches to speed up MC simulations of polyelectrolytes in low dielectric solvents, namely clothed global moves (Section 3) and parallel expanded ensembles (Section 4).

The use of clothed global moves for polyelectrolytes was first suggested by Gordon and Valleau [4] for the pivot move of a polyelectrolyte with explicit counterions. Gordon and Valleau used the primitive model to study a polyelectrolyte with monovalent counterions in aqueous solutions. For systems with strong electrostatic interactions considered here, much larger efficiency gains are found. Section 3 present results showing how the clothed pivot increases the speed-up from about two for $\epsilon=78$ to 400 for $\epsilon=24$.

An alternate approach to the study of strongly interacting systems is to use expanded ensembles techniques [11]. The idea is to create an extra simulation dimension (here the dielectric constant) which provides a route to circumvent high barriers in the free energy landscape, without having to climb over them directly. By running simulations at different values of $\epsilon$ on 
different computing nodes an effective parallel algorithm is constructed [5]. The simulations conducted at higher $\epsilon$ will converge fast while those at lower $\epsilon$ may become trapped in local minima in the free energy landscape. However, by allowing the different simulations to communicate and exchange conformations, those configurations trapped in low $\epsilon$ runs may escape via the higher $\epsilon$ runs. Also, if variations along the extra dimension are of interest, additional results may be accumulated at no extra effort. The results of Section 4 show how an expanded ensemble algorithm scales efficiently on parallel hardware at least to 8 processors.

\section{The model and simple Monte-Carlo method}

The basic system consists of a single polyelectrolyte with a total number of monomers $N$. The polyelectrolyte is modelled as a chain of identical hard sphere monomers each carrying a charge $q_{m}$. Each monomer is connected to its neighbour by a rigid but freely rotating bond of fixed length $b$. The polymer is enclosed in a spherical cell, with radius $R_{c}$, and the middle monomer is fixed at the centre of the cell. Also in the cell are hard sphere counterions; $N_{c}$ is the number of neutralising counterions each with charge $q_{c}$. In the cell model, only interactions within the cell are accounted for and no particles are allowed to escape the cell. All simulations are carried out in the so-called Primitive Model whereby the solvent is described by the dielectric constant $\epsilon$.

The Hamiltonian for the system is the sum

$$
U=U_{\mathrm{el}}+U_{\mathrm{hc}}+U_{\text {cell }},
$$

where the electrostatic term

$$
U_{\mathrm{el}}=\sum_{i<j}^{N+N_{c}} \frac{q_{i} q_{j} e^{2}}{4 \pi \epsilon \epsilon_{0}\left|\mathbf{r}_{\mathbf{i}}-\mathbf{r}_{\mathbf{j}}\right|},
$$


and $e$ is the elementary charge, $\mathbf{r}_{i}$ is the coordinate and $q_{i}$ is the valence of particle $i, U_{\mathrm{hc}}$ is a hard core potential preventing the particles from getting closer than a distance $d$ (the hard sphere diameter) from each other, and $U_{\text {cell }}$ is the term that confines all ions and monomers to reside within the spherical cell of radius $R_{c}$. In our simulations the following parameters are used: $d=4 \AA$ and $b=6 \AA$. The monomers have a charge of $q_{m}=+1$ and the counterions have a charge $q_{c}=-1,-2,-3$ or -4 and obeys the electroneutrality condition, $N q_{m}+N_{c} q_{c}=0$.

Simulations are carried out in the canonical ensemble and new monomer configurations are generated by pivot moves [12] and the small ions are translated. A pivot move involves choosing a monomer, between 2 and $N$, and a rotation angle, between 0 and $\delta_{\text {rot }}$. In all simulations $\delta_{\text {rot }}=360^{\circ}$. The chosen monomer divides the polymer in two and the smaller part of the polyelectrolyte is rotated. This ensures that the middle monomer is kept fixed. Energies are computed from Equation 1 and moves are accepted according to the Metropolis MC scheme [1] with the acceptance probability $p=\min [1, \exp (-\beta \Delta U)]$ where $\Delta U=U($ new $)-U($ old $)$ and $\beta=1 / k_{B} T$, with $k_{B}$ being the Boltzmann constant. $U$ (new) and $U$ (old) are the energies for the new and old configuration. The autocorrelation for the end-to-end distance is calculated as

$$
c_{R_{\mathrm{ee}}}(t)=\left\langle R_{\mathrm{ee}}(0) R_{\mathrm{ee}}(t)\right\rangle=\frac{1}{t_{\mathrm{max}}} \sum_{t_{0}=1}^{t_{\mathrm{max}}} R_{\mathrm{ee}}\left(t_{0}\right) R_{\mathrm{ee}}\left(t_{0}+t\right) .
$$

Here the time is discretised and the averaging is performed over $t_{\max }$ time origins. We also define the integrated autocorrelation time

$$
\tau=\int_{0}^{\infty} c_{R_{\mathrm{ee}}}(t) d t .
$$

$\tau$ is used as a measure of how fast a certain property of the simulation converges. In practise the integration is not drawn to infinity but to a point where the integrated autocorrelation does not increase any more. The integrated autocorrelation time $\tau$ gives a measure of how long a simulation has 
to be run before statistically independent values of a certain property can be found. Thus, shorter autocorrelation times will allow us to collect good statistics faster and the simulation converges in shorter time. Further details of the simulation model and method can be found in previously reported studies dealing primarily with DNA condensation $[10,9]$.

\section{Clothed global moves}

The pivot move is far superior to simple translations of individual monomers. For neutral polymers and polyelectrolytes with screened coulomb interactions, chains with many thousand monomers are run on relatively modest hardware $[12,8]$. When explicit ions are introduced, which is necessary for studying strong electrostatic interactions, two problems arise:, pivot trial moves can be rejected due to hard core interactions with the small ions; and pivot trial moves can be rejected due to a large energy difference when a large part of the chain is moved out of its small ion atmosphere.

In the presence of many small particles, for instance when salt is added, the first problem becomes the limiting factor while the second is dominant for the case of large electrostatic interactions. In both cases, the moves involving a big part of the chain are those most likely to be rejected.

The idea behind the clothed pivot move is to include part or all of the small ions surrounding the polyelectrolyte in the global move. By doing so the energy changes involved in the move will be smaller and the acceptance rate will be higher and this allows pivoting through larger angles.

The clothed pivot moves are performed as described in Section 2, except that ions within a distance $h$ from monomers involved in the pivot are moved with the chain with a probability $p_{c}$. To ensure detailed balance, the acceptance probability must be changed to [4]

$$
p=\min \left[1,\left(1-p_{c}\right)^{\Delta m} \exp (-\beta \Delta U)\right],
$$


where $\Delta m$ is the difference, before and after the clothed move, in the number of ions within the distance $h$ from monomers involved in the pivot move. The two parameters $h$ and $p_{c}$ are input to the simulations and affect the efficiency of the simulation in the same way as the choice of pivot angle parameter $\delta_{\text {rot }}$ or small ion translation distance parameter $\delta_{\text {tr }}$. From our experience and others [4], the choice of $h$ is not sensitive except that $h$ should include the ions closely correlated to the chain. Here we make the empirical choice: $h=3 d$. The choice of $p_{c}$ depends on the strength of the electrostatic interactions. For the case of monovalent counterions in water $(\epsilon=78)$ Gordon and Valleau [4] found $p_{c}=0.75$ to be optimal, whereas for trivalent counterions or low dielectric constant $(\epsilon<20) p_{c}=1$ appears to be the best choice. In between the clothed global moves, it is also important to relax the counterion configurations by moving them individually. In line with earlier work [10] we choose to perform attempted moves on half of the ions between every global move.

A common measure of conformational properties for polymers is the endto-end distance $R_{\mathrm{ee}}$. In order to illustrate how $R_{\mathrm{ee}}$ converges, the autocorrelation of $R_{\mathrm{ee}}$ is shown in Figure 1. For the water case of $\epsilon=78$ there is no difference between the clothed and the simple algorithm for the short $N=32$ chain for which the end-to-end distance decorrelates in only $2 \mathrm{MC}$ iterations. For lower dielectric constants of $\epsilon=39$ and $\epsilon=24$ there is a very significant speed-up in runs carried out with the clothed pivot algorithm. These results are quantified in Table 1 where speed-up factors of over 400 are attained for $\epsilon=24$.

Another way of increasing the electrostatic interaction is to include multivalent counterions in the system. Polyelectrolytes together with multivalent counterions have attracted significant interest; for example, it is well known that multivalent polyamines are responsible for DNA condensation [2]. Figure 2 shows the autocorrelation times for polyelectrolytes with counterions of different charges. As expected, longer chains $(N=292)$ converge slower than shorter chains $(N=24)$. The convergence is also slower with increasing 


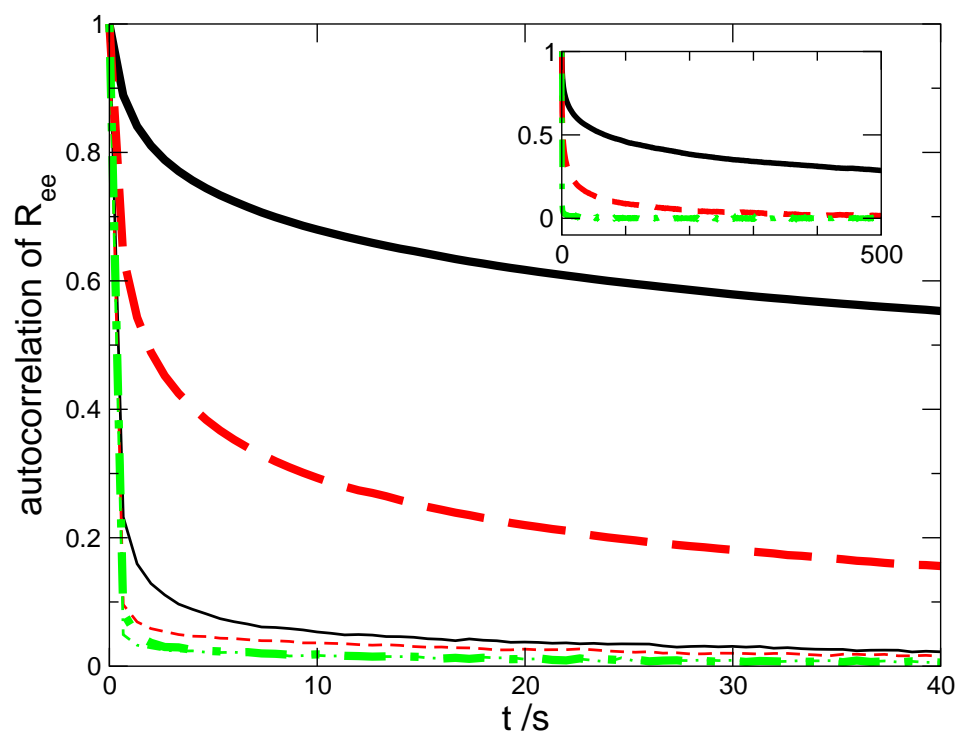

Figure 1: The autocorrelation of $R_{\text {ee }}$ for a polyelectrolyte with $N=32$ and monovalent counterions at different dielectric constants, $\epsilon=24$ (solid black line), $\epsilon=39$ (dashed red line), and $\epsilon=78$ (dot-dashed green line). The thin lines are from simulations performed with the clothed pivot algorithm and the thick lines are from simulations with the simple pivot. The inset shows the autocorrelation curves obtained with the simple pivot for a larger number of MC iterations. In the main figure the two dot-dashed lines are on top of each other. 
TABLE 1: Integrated autocorrelation times for the original Monte-Carlo algorithm $\tau_{\mathrm{MC}}$, for the clothed pivot algorithm $\tau_{\mathrm{cp}}$ and for the parallel $\mathrm{P} \epsilon$ method $\tau_{\mathrm{P} \epsilon}$ with $n_{\text {procs }}=8(\epsilon=24$ to 78$)$. $A$ is the percentage of accepted swaps between a certain $\epsilon$-ensemble and its nearest neighbours. Note that no speed-up is found for high $\epsilon$ since the resolution was not sufficient to distinguish between them as they decorrelate in only $2 \mathrm{MC}$ iterations.

\begin{tabular}{|c|cccccc|}
\hline$\epsilon$ & $\tau_{\mathrm{MC}}(\mathrm{s})$ & $\tau_{\mathrm{cp}}(\mathrm{s})$ & $\tau_{\mathrm{MC}} / \tau_{\mathrm{cp}}$ & $\tau_{\mathrm{P} \epsilon}(\mathrm{s})$ & $\tau_{\mathrm{MC}} / \tau_{\mathrm{P} \epsilon}$ & $A(\%)$ \\
\hline 24 & 160 & 0.36 & 440 & 20 & 8.1 & 7.7 \\
28 & 45 & 0.34 & 132 & 8.6 & 5.2 & 7.8 \\
33 & 17 & 0.30 & 57 & 4.1 & 4.1 & 7.8 \\
39 & 4.0 & 0.26 & 15 & 1.8 & 2.2 & 8.1 \\
47 & 0.86 & 0.24 & 3.6 & 0.70 & 1.2 & 8.9 \\
55 & 0.35 & 0.22 & 1.6 & 0.28 & 1.3 & 11 \\
65 & 0.24 & 0.20 & 1.2 & 0.25 & 1.0 & 14 \\
78 & 0.14 & 0.15 & 1.0 & 0.14 & 1.0 & 16 \\
\hline
\end{tabular}

valance of the counterions. However, the improvement in the rate of convergence at high counterion valencies due to the use of clothed pivot moves is very significant. The multivalent counterions will be in close proximity to the monomers due to the strong electrostatics. Therefore, when a global pivot move is attempted without taking these multivalent ions along, it will likely result in large changes in the configurational energy. Thus such unclothed pivot moves will likely be rejected, which in turn leads to the slow convergence of conformational properties.

In Figure 2, the time is shown as real time in seconds as run on a $1400 \mathrm{MHz}$ AMD Athlon Linux workstation. To show the time in terms of MC iterations would be misleading when discussing the clothed algorithm, since the decreased number of MC steps needed for convergence is counterbalanced by the increased number of pair interactions calculated in every move, since more particles are involved in the clothed pivot move. The increase in computational time for every move in the clothed pivot scales as $N_{c}^{2}$. Thus the 

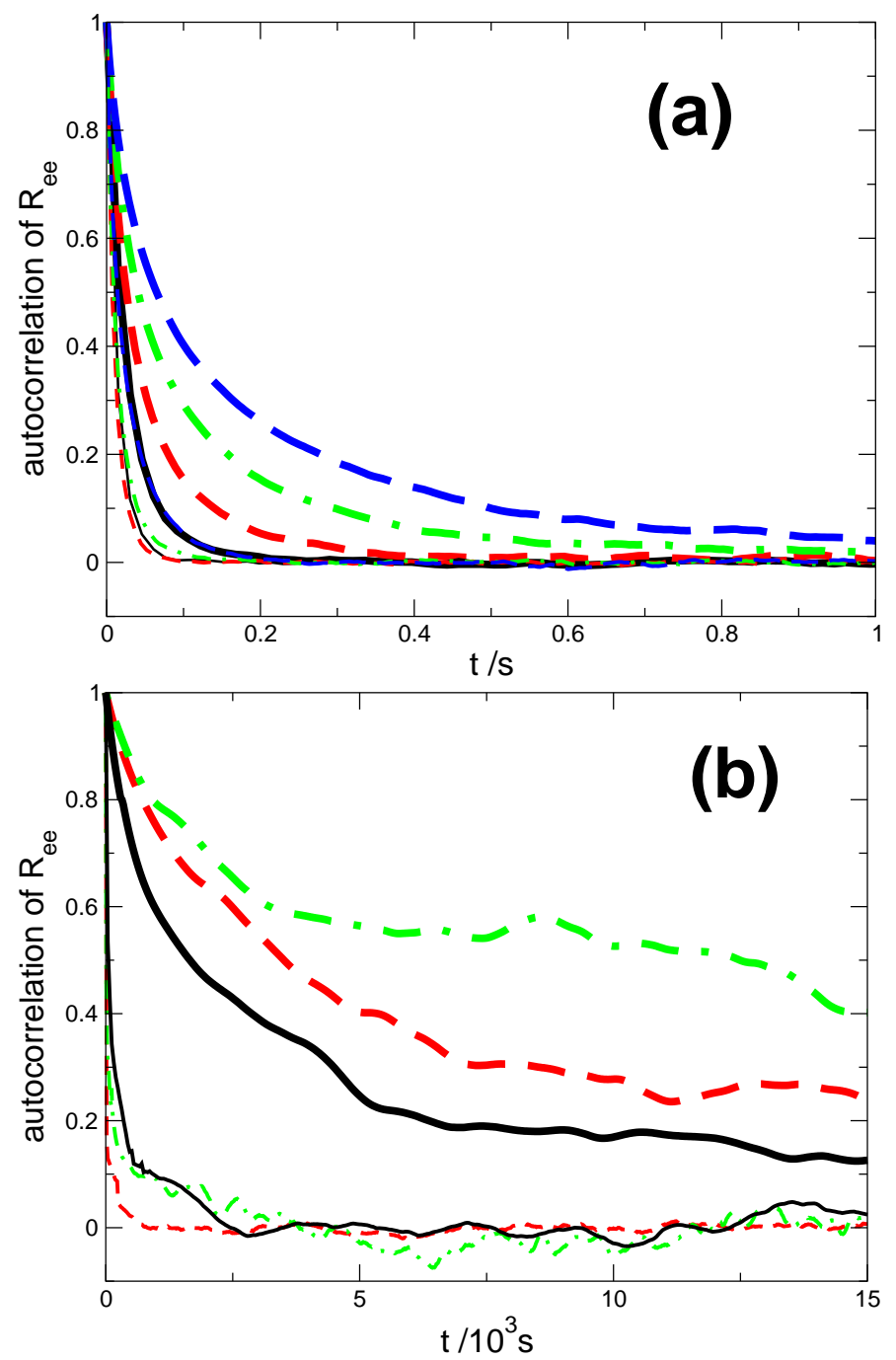

Figure 2: Autocorrelation for the end-to-end distance of polyelectrolytes with counterions of different valency: $q_{c}=1$ (solid black line), $q_{c}=2$ (dashed red line), $q_{c}=3$ (dot-dashed green line) and $q_{c}=4$ (long dashed blue line). The thick lines represent simulations performed with the simple pivot move while the thin lines come from simulations utilising the clothed pivot move. (a) $N=24$, note that the thin long dashed line is just on top of the thick solid line. (b) $N=292$. 
case with monovalent counterions will need 9 times longer for every move in comparison to the case with trivalent counterions, which is one reason why the algorithm only showed a 1.2 to 3 times efficiency improvement in the original work by Gordon and Valleau [4].

The proportion of accepted pivots as a function of the monomer at which the pivot is performed is plotted in Figure 3. For the simple pivot move see that pivot moves about those monomers that are located towards the middle of the chain are only accepted very infrequently. For the case with $q_{c}=3$ this difference is even more accentuated. Applying the clothed pivot move increases the percentage of accepted moves that involve the middle monomers. Accepted moves that involve larger portions of the chain lead to faster convergence of configurational properties. It is interesting to see that, when the clothed pivot is used, the case with $q_{c}=3$ has a flatter acceptance curve than for the case with $q_{c}=1$ and that the moves involving many monomers $(>30)$ are actually more often accepted for the trivalent case in comparison with the monovalent case. This flat curve resembles that of an uncharged polymer which suggests that the relative acceptance percentage of clothed pivot moves of a polyelectrolyte with tightly bound counterions is similar to that of simple pivot moves for a neutral polymer.

\section{Parallel expanded ensembles}

In this section we examine the application of the parallel ensemble method [5] to simulations of polyelectrolytes in low dielectric solvents. For the present problem it is natural to consider the expanded ensemble with $\epsilon$ as the parameter. Our implementation is a modification of the algorithm used in parallel tempering (PT) simulations of polymers by Irbäck and Sandelin [7] and we refer to it as $\mathrm{P} \epsilon$.

The $\mathrm{P} \epsilon$ algorithm consists of running multiple replicas of the system simultaneously at different $\epsilon$, where each replica is updated independently 


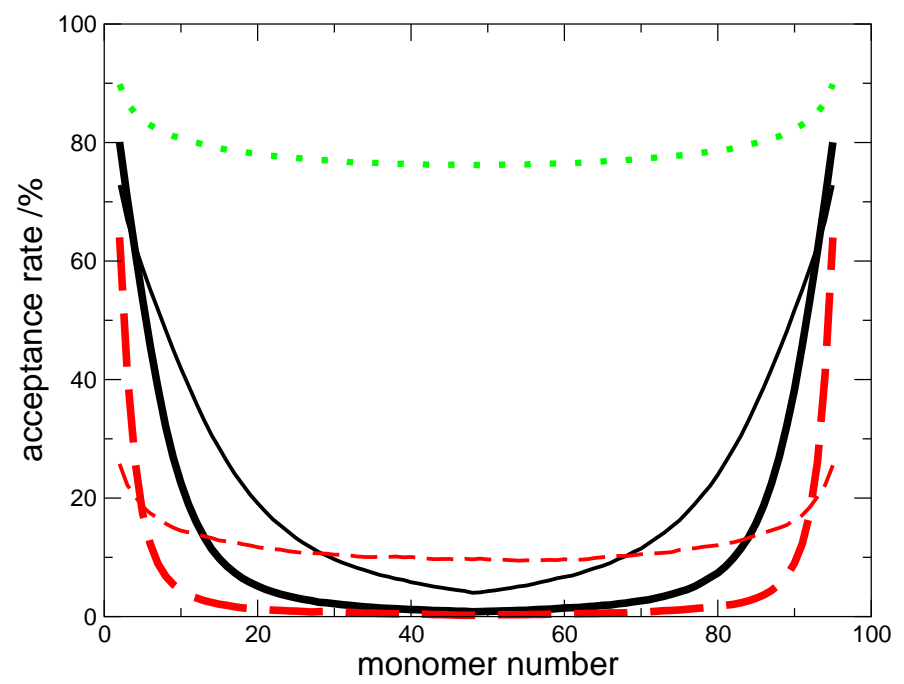

FiguRE 3: The acceptance rate of the pivot move as a function of monomer number. The polyelectrolyte has $N=96$ and monomer number 48 is fixed in the middle of the cell. The different curves correspond to systems with monovalent counterions (solid black lines), trivalent counterions (dashed red lines) and an uncharged polymer (dotted green line). Thick lines are from simulations with the simple pivot move and thin lines are from simulations with the clothed pivot. 
using the Metropolis Monte-Carlo technique described in Section 2. The implementation adopted here compares a pair of replicas, with neighbouring $\epsilon$, every 10 pivot moves to determine whether to exchange the polymer coordinates between the replicas. This was determined to give the best performance according to trial and error testing. This technique is suitable for parallelisation because communication between replicas does not occur often and the amount of information to be shared is small. Thus communication between systems takes very little time in comparison to other parts of the MC algorithm. Coordinate exchanges are accepted according to the Metropolis criterion [3] with probability

$$
p=\min \{1, \exp [-\Delta(\beta U)]\},
$$

where $\Delta(\beta U)=\Delta \beta \times \Delta U$ and the $\Delta$ refers to a comparison between two different replicas. Equation 6 is general for parallel expanded ensembles. Comparing two replicas $(a$ and $b$ ) in the $\mathrm{P} \epsilon$ ensemble gives

$$
p=\min \left\{1, \exp \left[-\beta\left(\frac{1}{\epsilon_{a}}-\frac{1}{\epsilon_{b}}\right)\left(U_{a}-U_{b}\right)\right]\right\} .
$$

The distribution of different $\epsilon$ over the total number of replicas $n$ is an input to the algorithm and affects the acceptance rate of exchanges and thus the efficiency of the method. A useful acceptance rate is a figure which should be high enough such that the algorithm is not effectively running $n$ completely independent MC simulations and yet still low enough such that all $n$ simulations do not evolve as a single MC simulation, using the same coordinates across $n$ replicas. Also, the distribution of different $\epsilon$ should be made such that the acceptance rates is the same over the different neighbouring pairs. This involves having larger gaps for large $\epsilon$. Following recipes given in the literature for the PT algorithm [6], we choose the distribution of $\epsilon$ in an exponential fashion according to

$$
\epsilon_{k}=\epsilon_{\min }\left(\frac{\epsilon_{\max }}{\epsilon_{\min }}\right)^{k /(n-1)}
$$


where $k$ refers to the replica number. Normally $\epsilon_{\min }$ will be determined by the physical problem. As a empirical rule of thumb an acceptance rate of between $10 \%$ and $20 \%$ for the exchanges between replicas appears to be optimal and $n$ and $\epsilon_{\max }$ should be chosen to satisfy this condition and any hardware constraints such as the available number of nodes.

To illustrate the efficiency of this method, a polyelectrolyte of length $N=$ 32 is selected and only monovalent counterions are present in the cell. Taking into consideration our computational limitations (typically 8 processors were available) and the need to achieve a useful acceptance rate of coordinate exchanges between replicas, we chose $\epsilon_{\min }=24$ and $\epsilon_{\max }=78$.

The end-to-end autocorrelation function is shown in Figure 4a for a few different values of $\epsilon$. For cases with high $\epsilon$, there is little difference between $\mathrm{P} \epsilon$ simulations compared to the serial algorithm. For smaller $\epsilon$ though, the simulations performed with the parallel algorithm have smaller correlation times than the corresponding simulations performed with the serial method. This is further emphasised in Table 1, where integrated correlation times are given for different $\epsilon$.

Even in the case where only results for $\epsilon_{\min }=24$ is of interest, linear speed-up can be achieved with this method. That is, by running the simulation on 8 processors it will take $1 / 8$ th the time of the serial method. If all $\epsilon$ values are of interest the total speed-up is $1.4\left(8 \times \tau_{p \epsilon}\left(\epsilon_{k}=24\right) / \sum_{k} \tau_{\mathrm{MC}}\left(\epsilon_{k}\right)\right)$, and thus super-linear speed-up has been achieved. The same scaling is not observed for higher $\epsilon$ and for $\epsilon=33$ the efficiency is 0.7 .

The rule to find the different $\epsilon$-ensembles and distribute them over processors is given by Equation 8. As mentioned above the number of accepted exchanges is an important quantity when designing the algorithm. In Table 1, the number of accepted trial exchanges $A$ between neighbouring replicas is given. $A$ goes from $8 \%$ for $\epsilon=24$ to $16 \%$ for $\epsilon=78$. This number directly reflects the degree of energy overlap between the different ensembles as shown in Figure 4b. 
(a)

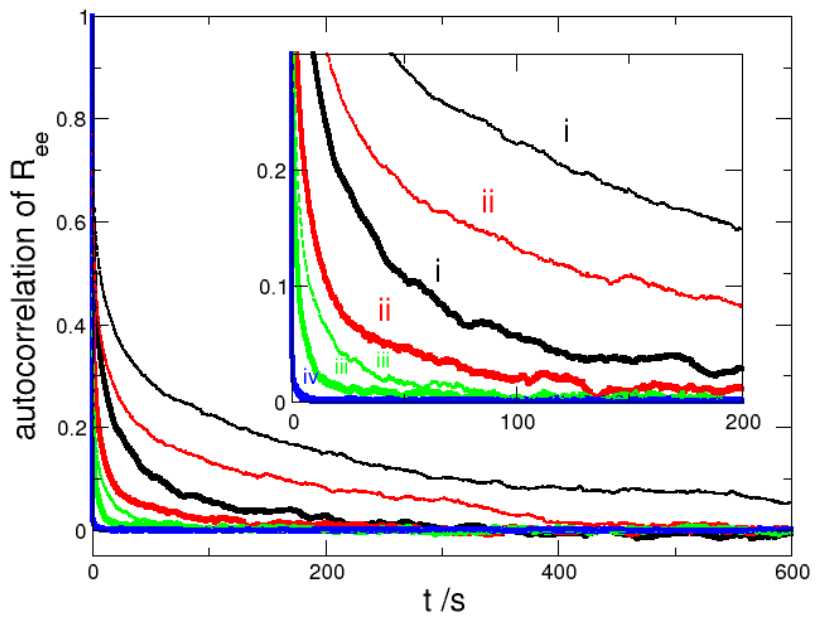

(b)

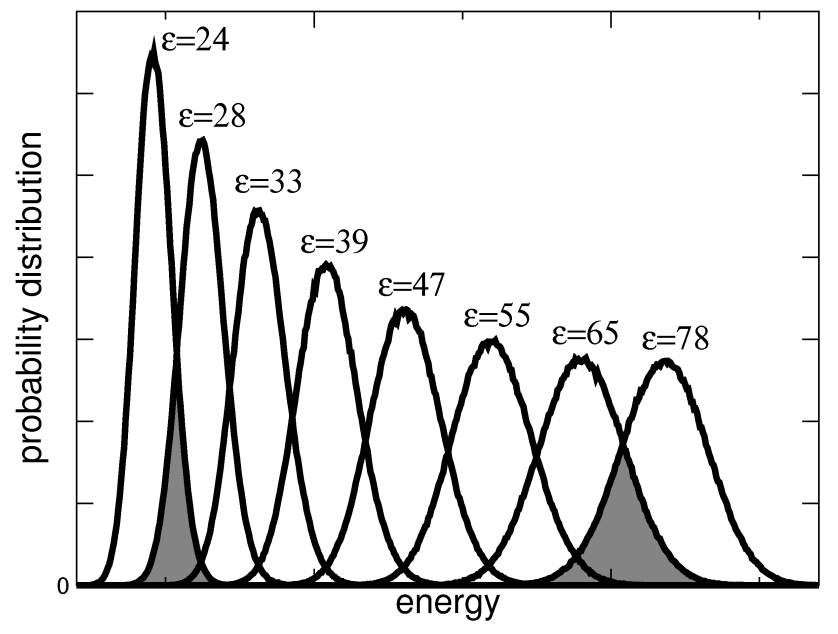

Figure 4: (a) The autocorrelation of $R_{\mathrm{ee}}$ for a polyelectrolyte with $N=32$ and monovalent counterions at different dielectric constants, from top to bottom $\epsilon=24$ (i), 28 (ii), 39 (iii) and 78 (iv). The thin lines are from serial MC simulations while the thick lines are from $\mathrm{P} \epsilon$ simulations performed on $n=8$ CPUs with $\epsilon_{\min }=24$ and $\epsilon_{\max }=78$. No clothed moves are used, that is, $p_{c}=0$. The inset is a blow-up for small $t$. (b) Probability distributions of the energy for the different replicas in a $\mathrm{P} \epsilon$ simulation with the same parameters as in (a). The gray areas marks the area overlap of the $\epsilon=24$ and $\epsilon=28$ curves, and the $\epsilon=65$ and $\epsilon=78$ curves. 
The energy overlap, pictured in Figure 4b, illustrates key points of the $\mathrm{P} \epsilon$ algorithm. First of all, only nearest neighbour ensembles have overlapping energies and thus other attempted exchanges are unlikely to be accepted. Further, the same energy overlap is preferable for all neighbouring $\epsilon$, since having the same acceptance rate for all $\epsilon$ would increase the efficiency of the method. Thus the simulation scheme can be improved by finding an alternative to Equation 8 that will result in all energy overlaps being of the same size.

\section{Conclusion}

We have given quantitative examples of how the clothed pivot algorithm and the parallel ensemble technique speed up simulations of single chain properties of polyelectrolytes when the electrostatic interactions are large; for example, when the solvent has a low dielectric constant.

The clothed pivot algorithm will always improve the convergence time for conformational properties when compared to the simple pivot; with the gains being more significant when multivalent counterions are present, or when the solvent has a low dielectric constant. This improvement is due to the lowering of the energy differences when performing global moves. Adding salt will pose additional problems due to hard core overlaps, but this method should be able to accommodate moderate salt concentrations.

The parallel ensemble technique can, if designed properly, give a high throughput of polyelectrolyte simulations. Linear speed-up can be found for systems with a large electrostatic coupling. Since the amount of communication is small, the parallel ensemble technique is suitable for cheap commodity hardware and cluster solutions. 
Acknowledgments: This work was supported by the Australian Research Council, the Victorian Partnership for Advanced Computing and the WennerGren Foundation.

\section{References}

[1] M. P. Allen and D. J. Tildesley. Computer Simulation of Liquids. Oxford University Press, Oxford, 1989. C1258

[2] V. A. Bloomfield. DNA condensation by multivalent cations. Biopymers, 44:269-282, 1997. C1260

[3] Bálint Joó et al. Parallel tempering in lattice QCD with O(a) improved Wilson fermions. Phys. Rev. D, 59:114501-114509, 1999. C1266

[4] H. L. Gordon and J. P. Valleau. A partially clothed pivot algorithm for model polyelectrolyte solution. Mol. Sim., 14:361-379, 1995. C1256, C1259, C1260, C1264

[5] Ulrich H.E. Hansmann. Parallel tempering algorithm for conformational studies of biological molecules. Chem. Phys. Lett., 281:140-150, 1997. C1257, C1264

[6] Ulrich H.E. Hansmann and Y. Okamato. Numerical comparisons of three recently proposed algorithms in the protein folding problem. J. Comput. Chem., 18:920-933, 1997. C1266

[7] Anders Irbäck and Erik Sandelin. Monte-Carlo study of the phase structure of compact polymer chains. J. Chem. Phys., 110(2):12256-12262, 1999. C1264

[8] B. Jönsson, C. Peterson, and B. Söderberg. Variational approach to the structure and thermodynamics of linear polyelectrolytes with 
coulomb and screened coulomb interactions. J. Phys. Chem., 99:1251-1266, 1995. C1259

[9] M. O. Khan and D. Y. C. Chan. Monte-Carlo simulations of stretched charged polymers. J. Phys. Chem. B, 107:8131-8139, 2003. C1259

[10] M. O. Khan and B. Jönsson. Electrostatic correlations fold DNA. Biopolymers, 49:121-125, 1999. C1255, C1259, C1260

[11] A. Lyubartsev, A. A. Martsinovski, S. V. Shevkunov, and P. N. Vorontsov-Velyaminov. New approach to Monte-Carlo calculation of the free-energy - method of expanded ensembles. J. Chem. Phys., 96:1776-1783, 1992. C1256

[12] N. Madras and A. D. Sokal. The pivot algorithm: A higly efficient Monte-Carlo method for the self-avoiding walk. J. Stat. Phys., 50:109-186, 1988. C1258, C1259

[13] M. J. Stevens and K. Kremer. The nature of flexible linear polyelectrolytes in salt free solution: A molecular dynamics study. J. Chem. Phys., 103:1669-1690, 1995. C1255

[14] M. Ullner. Polyelectrolyte models in theory and simulation. In S. Tripathy, J. Kumar, and H. S. Halwa, editors, Handbook of Polyelectrolytes and their Applications, Los Angeles, 2002. American Science. C1255

[15] R. G. Winkler, M. Gold, and P. Reineker. Collapse of polyelectrolyte macromolecules by counterion condensation and ion pair formations: A molecular dynamics study. Phys. Rev. Lett., 80:3731-3734, 1998. C1255 